\title{
Neutron and Photon Transport in Sea-Going Cargo Containers
}

J. Pruet, M. A. Descalle, J. Hall, B. Pohl, S. G. Prussin

September 16, 2004

Journal of Applied Physics 
This document was prepared as an account of work sponsored by an agency of the United States Government. Neither the United States Government nor the University of California nor any of their employees, makes any warranty, express or implied, or assumes any legal liability or responsibility for the accuracy, completeness, or usefulness of any information, apparatus, product, or process disclosed, or represents that its use would not infringe privately owned rights. Reference herein to any specific commercial product, process, or service by trade name, trademark, manufacturer, or otherwise, does not necessarily constitute or imply its endorsement, recommendation, or favoring by the United States Government or the University of California. The views and opinions of authors expressed herein do not necessarily state or reflect those of the United States Government or the University of California, and shall not be used for advertising or product endorsement purposes. 


\title{
Neutron and Photon Transport in Sea-Going Cargo Containers
}

\author{
J. Pruet, M.-A. Descalle, J. Hall, and B. Pohl \\ Lawrence Livermore National Laboratory \\ S. G. Prussin \\ Department of Nuclear Engineering, \\ University of California at Berkeley
}

(Dated: September 14, 2004)

\begin{abstract}
Abstact
Factors affecting sensing of small quantities of fissionable material in large sea-going cargo containers by neutron interrogation and detection of $\beta$-delayed photons are explored. The propagation of variable-energy neutrons in cargos, subsequent fission of hidden nuclear material and production of the $\beta$-delayed photons, and the propagation of these photons to an external detector are considered explicitly. Detailed results of Monte Carlo simulations of these stages in representative cargos are presented. Analytical models are developed both as a basis for a quantitative understanding of the interrogation process and as a tool to allow ready extrapolation of our results to cases not specifically considered here.
\end{abstract}

PACS numbers: 89.20.Dd, 89.40.Cc, 25.85.Ec 


\section{INTRODUCTION}

The detection of kilogram quantities of fissionable materials that might be hidden in large, sea- going cargo containers has been recognized as a major issue in world security. This represents a daunting task. Such intermodal cargo containers, with cross sections of about $2.4 \mathrm{~m} \times 2.4 \mathrm{~m}$ and lengths of $6 \mathrm{~m}$ or $12 \mathrm{~m}$, carry cargos of up to 27 metric tonnes. Essentially all materials in modern commerce, and hence elements spanning most of the periodic table, can be found among them. The contents of a container may be almost homogeneous in both composition and volumetric distribution, or heterogeneous, with quasi-regular or random voids throughout. For such a wide range of conditions, bruteforce Monte Carlo simulations of the transport of interrogating radiation and the subsequent production and transport of signature radiation through the cargo to a detector are the primary means of approaching reasonably accurate predictions of the efficacy of proposed methodologies. Performing these simulations for all possible cargos is impractical. It is of obvious interest to identify and quantify those factors that can be reduced to generalizations and to develop analytical approaches that can provide reasonable and prompt estimates of a given interrogation scenario.

Formalisms describing neutron and photon transport have long been in a state of mature development. Publications of the reactor physics community, in particular, are a rich resource for understanding many aspects of the cargo interrogation problem. However, the focus and interesting aspects of reactor applications are quite different from those for cargo interrogation systems. The present work is an attempt to bridge some of that disparity by developing part of the foundation needed for understanding cargo interrogation methods that rely on neutron-induced fission and detection of post-fission, high-energy $\beta$-delayed $\gamma$ rays. Figure 1 gives a simple illustration of such a detection system. We will emphasize the salient features of particle transport relevant to this problem.

The problem we deal with is defined principally by the extensive shielding posed by the massive cargos, the physical dimensions of the containers and the time allowed for an inspection, and decay imes of interesting radiation. The methodology chosen for study here is suggested by the fairly large mean free paths for neutrons with energies in the range $0.1 \leq E_{n} \leq 14 \mathrm{MeV}$ in most materials (see, e.g. [1]) and the high-penetrability and characteristic signatures of the $\gamma$-rays emitted with energies $E_{\gamma} \gtrsim 3 \mathrm{MeV}$ in the decay 
of short-lived fission products [2]. Practical considerations suggest that the time scale for scanning of cargo containers should be on the order of $1 \mathrm{~min}$, which is just the time scale associated with the decay of the fission products that emit photons of interest.

The estimation of the $\gamma$-ray signature escaping the cargo and incident upon an external detector can be treated in two distinct phases. The first concerns the interrogating neutrons, their transport through the cargo container and their efficiency for inducing fission. This is treated in Section 2. The second part concerns the high-energy $\gamma$-rays from decay of the fission products, their production, transport through the cargo container and their probability of detection. Here we consider a schematic scintillation device as the detector for such radiation. In section 3 we present estimates for the spectra of $\gamma$-rays emitted following fission as well as formulas for simply estimating the influence of cargo on the observed photon spectrum. As representative of possible clandestine material, we treat explicitly the case of highly-enriched uranium (HEU) embedded in homogeneously-filled containers. Effects due to the environment outside the detector/container system, including scattering in the external environment, are neglected. To represent common hydrogenous materials, such as plastics, foodstuffs and all other organic products and materials, we choose a model composition for wood. To represent the most common heavier elements found in commerce, we consider aluminum and steel. Load limits generally applied to these containers dictate a maximum mean cargo density of $\rho_{\max } \approx 0.6 \mathrm{~g} \mathrm{~cm}^{-3}$.

\section{NEUTRON TRANSPORT IN CARGOS AND FISSION}

One key measure of the usefulness of active interrogation systems is the neutron flux at the position in the cargo container where a target may be located. This flux will depend primarily on the properties of the cargo and the characteristics of the interrogating neutron beam. At the start, it is useful to differentiate between cargos that strongly or weakly moderate the neutrons. Practically, this is equivalent to distinguishing between materials rich in hydrogen and those that are not[10]. The first case, i.e. transport in weakly-moderating cargos, is relatively straightforward because thermal energies are generally not reached and loss of neutrons by absorption is usually negligible. To a good approximation, only scattering of quasi mono-energetic neutrons need be considered. This case is discussed first. 


\section{A. Weakly Moderating Cargos}

Cargos composed only of heavy elements are not efficient moderators because of the small energy loss characterizing neutron collisions within the container and the relatively few collisions suffered before neutrons escape the container. In the limit of energy-independent neutron scattering, adequate for rough scaling purposes, the mean distance traversed by a neutron after scattering $n$ times in a homogeneous medium is $\left\langle r^{2}\right\rangle=2 n \lambda^{2}$, where $\lambda$ is the scattering mean free path. For a cargo container with characteristic linear dimension $L$, the number of scatterings suffered by a typical neutron before escaping the container is

$$
n_{\mathrm{esc}} \approx \frac{1}{2}\left(\frac{L}{\lambda}\right)^{2} .
$$

A simple estimate of the upper limit to $n_{\mathrm{esc}}$ can be found by neglecting compound elastic scattering and using the low-energy limit to potential scattering

$$
\sigma_{e l, p o t}=4 \pi R^{2}
$$

Here $R \approx 1.25 A^{1 / 3}$ fermi is the nuclear radius and $A$ is the mass number of the target nucleus. With this, $\lambda \approx 8.5 \mathrm{cmA}^{1 / 3} / \rho$ and an estimate of the number of collisions to escape is

$$
n_{\mathrm{esc}} \leq 7 \cdot 10^{-3}\left(\frac{\rho L}{A^{1 / 3}}\right)^{2},
$$

where $\rho$ is the cargo density. The average number of collisions needed to thermalize a neutron with initial energy $E_{0}$ is about

$$
n_{\mathrm{th}} \approx \frac{\ln \left(E_{0} / E_{t h}\right)}{\xi}
$$

where

$$
\xi=\frac{d E}{E} \approx \frac{2}{A+2 / 3}
$$

is the mean logarithmic energy loss per collision. If we assume the maximum homogeneous density of $\rho_{\max }=0.6 \mathrm{~g} \mathrm{~cm}^{-3}$, the number of collisions suffered by a neutron before traversing a distance $\mathrm{L}$ in a container filled with either aluminum or steel will be less than about 16 and 10, respectively. For the minimum neutron source energy of $0.06 \mathrm{MeV}$ considered here, the ratio of the number of collisions to escape to the number of collisions to thermalize is less than 0.1 for all mass numbers greater than about 25.

Inelastic scattering changes the evolution of neutron energy within the container a little. For incident neutron energies larger than a few $\mathrm{MeV}$, inelastic scattering comprises about 
half the total scattering cross section for many nuclei with $\mathrm{A} \gtrsim 20$. Each inelastic scattering event can decrease the neutron energy by a factor of $\sim 2$. Once the neutron reaches energies of a few hundred keV inelastic scattering is usually not efficient at moderating the neutron. At least for semi-quantitative calculations, thermalization can be neglected safely, as well as loss of neutrons by mechanisms other than escape from the container.

Assuming that neutron absorption and the energy dependence of the scattering cross section can be neglected, a simple diffusion approximation for neutron transport can be applied. That is, the total flux in the container is given approximately by the sum of the uncollided flux at the source energy (or energies) and a diffusive flux. We consider for simplicity the case of a mono-energetic neutron beam entering through the wall of a homogeneously filled container. The flux of uncollided neutrons along the beam axis in the cargo is given simply by

$$
\phi_{0}(z)=\phi_{0} e^{-z / \lambda}
$$

where $\phi_{0}$ represents the flux of neutrons at the point of entering the cargo container and $z$ represents the path length within the container of an uncollided neutron.

If scattering is approximately isotropic, as is typically true for modestly heavy nuclides, the neutrons undergoing a first scattering out of the beam can be treated as a source of diffusive flux. The number of neutrons scattered out of the beam at $z$ per unit time per unit volume is

$$
\frac{d S}{d^{3} x d t}=\Sigma \phi_{0}(z)
$$

Here $\Sigma=\sigma \rho N_{a} / A$ is the macroscopic scattering cross section and $N_{a}$ is Avogadro's number. The flux at an arbitrary point within the cargo container can be calculated from an estimate of the diffusion kernel that describes propagation of neutrons from a localized source. In an infinite and homogeneous medium, the diffusive flux at a point $\bar{r}^{\prime}=\left(x^{\prime}, y^{\prime}, z^{\prime}\right)$ arising from a point source of strength $d S$ at $\bar{r}=(x, y, z)$ is

$$
d \phi_{\mathrm{diff}}(r) \equiv \frac{d S}{4 \pi D} G_{\mathrm{inf}}\left(\bar{r}, \bar{r}^{\prime}\right) \approx \frac{d S}{4 \pi D\left|\bar{r}-\bar{r}^{\prime}\right|}
$$

Here $D$ is the familiar diffusion coefficient given by $D^{-1} \approx 3 \Sigma(1-\bar{\mu})$, with $\bar{\mu}$ is the average of the cosine of the scattering angle.

In finite containers the diffusive flux approaches a very small value near the container walls. Eq. 8 is only accurate when the distance $\left|\bar{r}-\bar{r}^{\prime}\right|$ is much smaller than the distance 
from $\bar{r}^{\prime}$ to the nearest container wall. Standard approximations to the diffusion kernel for rectangular boxes are well known, though somewhat tedious. Appendix 2 reviews some properties of the finite-space diffusion kernel $G_{\text {fin }}$. In terms of this kernel, a simple estimate of the diffusive flux at any point in the cargo container is given by

$$
\phi_{\text {diff }}(\bar{r})=\frac{\Sigma}{4 \pi D} \int \phi_{0}\left(z^{\prime}\right) G_{\text {fin }}\left(\bar{r}, \bar{r}^{\prime}\right) d^{3} r^{\prime}
$$

where $\bar{r}^{\prime}$ is the point of first scatter for a neutron.

For homogeneous cargos, the sum of the uncollided flux (eq. 6) and the diffusive flux (eq. 9) are usually adequate for predicting the total flux at a target to within a factor of about two in homogeneous cargos. To demonstrate this, and to motivate a simple picture describing interrogation of non-hydrogenous cargos, the neutron flux in a container filled homogeneously with natural aluminum is shown in figure 2 as a function of beam energy. Results from the above analytic calculations and from detailed Monte Carlo calculations are both shown. These Monte Carlo simulations are from the LLNL COG [3] code and use ENDF B-VI evaluated data files [11]. The neutron source was taken to be a $30 \mathrm{~cm}$ diameter beam incident normally at the center of the cargo container floor. The container walls were assumed to be low carbon steel of thickness $1 / 8^{\prime \prime}$. Above the floor was a $3 \mathrm{~cm}$ thick sheet of wood, modeled as cellulose $\left(\mathrm{C}_{6} \mathrm{H}_{10} \mathrm{O}_{5}\right)$ containing $12 \% \mathrm{H}_{2} \mathrm{O}$ by weight.

The comparisons between the simple analytical approximations and the Monte Carlo simulations show reasonable agreement. They are also representative of results for steel and other homogeneous non-hydrogenous cargos. Common to these is the relatively weak beam attenuation. For example, the flux of $14 \mathrm{MeV}$ neutrons at the center of a container filled at maximum mean density is still about $10 \%$ of the incident flux. Also, and in contrast to hydrogenous cargos (see below), the flux depends only modestly on the neutron beam energy. The wood flooring is responsible for most of the dependence of the flux on incident neutron energy. For example, the wood attenuates $60 \mathrm{keV}$ neutrons by a factor of about 3, and $1 \mathrm{MeV}$ neutrons by a factor of about two.

The results shown in figure 2 are relevant for the case of a neutron beam positioned directly beneath a target in a static condition. However, active interrogation systems now under consideration propose scanning the cargo by passing the container over or by the beam at a constant rate. For this case, it is important to understand the relative importance of both the uncollided and diffusive flux throughout the container. When the diffusive flux 
dominates, the fission rate at the target will vary relatively slowly as the container traverses through the beam. However, when the uncollided beam dominates, a signature from fission will increase markedly when the target enters the vicinity of the beam axis. Figure 3 shows the spatial variation of the total flux along the horizontal centerline of the cargo container for a $14.5 \mathrm{MeV}$ neutron beam. The cargo and beam geometries represented by this figure are the same as those described by figure 2. It is clear that the flux within the volume defined by the uncollided beam is about an order of magnitude larger than the flux $1 \mathrm{~m}$ away from the beam. This is typical of many weakly-moderating homogeneous cargos. The uncollided flux typically dominates over the diffusive flux because the container weight limit precludes mean free paths smaller than about $40(\mathrm{~A} / 27)^{-1 / 3} \mathrm{~cm}$. For homogeneous cargos, then, the fission signature will rise appreciably as the target enters the uncollided neutron beam. Alternatively, the makeup of the cargo outside of the region defined by the neutron beam will generally have little influence on the flux within this region.

\section{B. Cargos that Efficiently Moderate and Absorb Neutrons}

Neutron scattering on hydrogen is characterized by an average logarithmic energy loss $\xi=1$ for energies larger than a few times the average thermal energies of target protons $\left(\sim 10^{-1} \mathrm{eV}\right)$. Unlike the case of cargos composed of elements with large atomic mass, the container weight limit does not rule out thermalization of neutrons with energies of several $\mathrm{MeV}$. Indeed, because the n-p scattering cross section increases so quickly with decreasing neutron energy - the scattering cross section for $1 \mathrm{MeV}$ neutrons is about 12 times larger than for $14 \mathrm{MeV}$ neutrons - neutrons thermalize in common cases of hydrogen-rich cargos.

In the previous section we showed that consideration of the finite container size is important for estimating the offf-axis flux in inefficiently moderating cargos. Properly accounting for the influence of neutron leakage in efficiently moderating cargos is more difficult and would take us beyond the reach of simple integrals. However, we will show below that the finite container size plays a very modest role in setting the flux in hydrogenous cargos. This is because in these cargos the absorption length, i.e. the distance travelled by initially nonthermal neutrons before being thermalized an absorbed, is typically smaller than the cargo container size. The presence or absence of material outside of the absorption length can only have an exponentially small influence on the diffusive thermal flux. 
When neutron moderation is important, account must be taken of the neutron energy distribution and the dependence of the neutron cross section on energy. One useful and relatively simple approach to describe the neutron spectrum in moderating media is provided by Fermi age theory (see [4]). This theory directly considers the slowing down density of neutrons in the medium defined as

$$
q=\xi \Sigma E \frac{d \phi}{d E}
$$

Here $q$ is the slowing down density, or rate per unit volume at which neutrons with energy $E$ scatter to lower energies, and $d \phi / d E$ is the differential flux of neutrons with energy $E$. In time-independent systems the volume integral of $q$ is independent of $E$. For a point source of mono-energetic neutrons in a uniform medium, Fermi's theory gives a slowing down kernel described by the Gaussian form

$$
q(r, \tau)=\frac{e^{-r^{2} / 4 \tau}}{(4 \pi \tau)^{3 / 2}}
$$

Here $\tau$ is the so called Fermi age and is usually given in $\mathrm{cm}^{2}$. The quantity $\tau(E)$ determines the spatial width of the distribution of neutrons with energy $E$. In addition to being unwieldy for calculations, it is well known that a Gaussian kernel underestimates the slowing down density at large $r$ for water and water-like moderators characterized by $\xi \approx 1$. Instead of the Gaussian kernel, we use the simple exponential form

$$
q=\frac{\exp (-r / \sqrt{\tau(E)})}{4 \pi \tau(E) r}
$$

that is known empirically to represent the slowing down density at large $r$ for water and water-like moderators to a good approximation [5]. For the present calculations we rely on published values of neutron thermalization ages, i.e. $\tau\left(E=E_{\text {thermal }}\right)$ [5]. Table I gives transport quantities characterizing passage of neutrons through plywood.

Once neutrons reach near-thermal energies, scattering and absorption cross sections remain approximately constant. It then is appropriate to describe these near-thermal neutrons with a diffusion equation. Treating eq. (10) as the source in a diffusion equation, the diffusive thermal flux corresponding to a point source of unit strength of mono-energetic neutrons is

$$
\phi_{\text {diff,th }}=\frac{1}{4 \pi \tau D} \frac{1}{1 / \tau-k^{2}} \frac{1}{r} \exp (-k r)\left(1-e^{r\left(k-\tau^{-1 / 2}\right)}\right)
$$

where $\mathrm{D}$ is the diffusion coefficient for thermal neutrons and $\tau$ here represents the age of thermal neutrons. In eq. $13 k^{-1} \approx \sqrt{D / \Sigma_{a}}$ is the diffusion length for thermal neutrons, with 
$\Sigma_{a}$ the macroscopic absorption cross section of thermal neutrons. We note that in practical cases, some of the neutrons interrogating hydrogenous cargo will have scattered multiple times, but will not have thermalized. Eq. 10 suggests an estimate for the flux of these intermediate-energy neutrons. As a first approximation,

$$
d \phi_{\text {int }} \approx 2 \lambda_{0} d q\left(r, \tau=\tau_{\text {therm }}\right)
$$

where $\lambda_{0}$ is the mean free path of neutrons with the incident energy. We neglect these intermediate energy neutrons in the following because the rate at which they induce fission is typically small compared to the fission rate associated with thermal neutrons.

We again take the once-scattered neutrons as the source of aging/diffusing neutrons in eq. 7. This gives an estimate of the diffusive thermal flux at any point in the cargo container as

$$
\phi_{\text {diff }, \text { th }}(\bar{x})=\frac{\Sigma}{4 \pi \tau D} \frac{1}{1 / \tau-k^{2}} \int \frac{\phi_{0}\left(z^{\prime}\right)}{r\left(\bar{x}, \bar{x}^{\prime}\right)} \exp \left(-k r\left(\bar{x}, \bar{x}^{\prime}\right)\right),\left(1-e^{r\left(\bar{x}, \bar{x}^{\prime}\right)\left(k-\tau^{-1 / 2}\right)}\right) d^{3} x^{\prime} .
$$

where the integral is over the volume defined by the unscattered neutron beam.

To illustrate the general features of neutron interrogation of hydrogenous cargos, we have used the wood model described above, expected to be representative of a wide variety of agricultural products, plastics, etc. The neutron flux at the center of a container filled with this material is shown in figure 4 as a function of incident neutron energy for both Monte Carlo simulations and the analytic model developed here. Again, the fluxes calculated with the analytical model are in good agreement with those obtained from the Monte Carlo simulations. Both point out the central feature that the flux in thick hydrogenous media can depend markedly on the energy of incident neutrons. This reflects the strong energy dependence of the n-p scattering cross section. Neutrons with energies of $10-14 \mathrm{MeV}$ penetrate hydrogenous cargos about as well as they penetrate higher-Z cargos. However, at energies less than a few $\mathrm{MeV}$, strong attenuation occurs in the hydrogenous media. It is worth noting that for $E_{n} \lesssim 3 \mathrm{MeV}$, the fraction of the flux that is uncollided is negligible. In a container filled uniformly with our wood model at a density of $\rho=0.6 \mathrm{~g} \mathrm{~cm}^{-3}$, for example, the uncollided flux of incident $60-\mathrm{keV}$ neutrons at the center of the container is about 5 orders of magnitude smaller than the diffusive flux of thermal neutrons at the container center.

The spatial variation of the flux for this cargo is illustrated in figure 5. Like the distribution shown in figure 3 for aluminum, which is expected to be representative of most weakly 
moderating media, the diffusive flux in hydrogenous media decreases almost exponentially with distance normal to the beam axis. For hydrogenous, moderators, however, this arises not so much from the finite container size, but principally because of thermal neutron absorption. Because of the axial dependence of beam attenuation, iso-flux surfaces are not well-represented by hemi-spheres centered at the point in the floor where the beam enters the cargo container. Even though the mean free path of incident neutrons can be small relative to the cargo height, it is not appropriate to approximate the neutrons as suffering a first collision at their point of entry.

\section{SPECTRUM AND TRANSPORT OF $\beta$-DELAYED $\gamma$ RAYS IN CARGOS}

The neutron-rich primary fission fragments, after emission of prompt neutrons and $\gamma$ rays, have atomic numbers that are typically 3-4 units smaller than those corresponding to stability for the same mass number. As these nuclides undergo $\beta$ decay to reach stability, a large number of $\gamma$ rays are emitted. Figure 6 illustrates some of the processes that occur during such decays. At the present time, no single evaluated data set exists that directly provides for the temporal evolution of $\gamma$ rays from the decay of the fission products. However, evaluated data sets representing all of the physical parameters required for such calculations have been prepared. These include the independent and cumulative fission yields of all fission products, branching ratios in the decay of ground and isomeric states, lifetimes of these states, and the spectra of $\gamma$ rays emitted in their decay. Sizeable uncertainties and, possibly, significant errors are likely present for the shortest-lived fission products. High-energy $\gamma$ ray spectra generated from some of these data sets have been shown to be in reasonable agreement with initial experiments designed specifically to test them under conditions likely to be of interest to cargo interrogation $[2,6,7]$.

Of particular interest is the time evolution of the emission of the high-energy $\gamma$ rays produced following fission. Figure 7 illustrates the predicted time evolution of the emission of such photons following thermal fission of ${ }^{235} \mathrm{U}$ and ${ }^{239} \mathrm{Pu}$. These nuclides are expected to exhibit the general and generic features common to most fissile nuclides. The results shown here were obtained from Monte Carlo simulations using the fission yield evaluations of England and Rider [8] and the NUDAT data files [9]. As seen in the figure, about half of the high-energy photons, $\approx 0.05-0.1$ per fission, are emitted within about 10 sec 
following fission and most of the remainder are emitted over a period of order 100 sec. The total intensity of photons with $E_{\gamma} \gtrsim 3 \mathrm{MeV}$ is roughly an order of magnitude larger than the intensity of $\beta$-delayed neutrons, the signature of fissile nuclides often used to detect fissionable material under conditions of minimal shielding.

Figure 8 shows details of the photon spectrum accompanying thermal fission of ${ }^{235} \mathrm{U}$. Although a number of prominent and identifiable lines are present in the spectrum, about half of the total intensity is found in a large number of fairly weak lines. While this complicates attempts at comparing calculations with experiments, it also suggests the efficacy of the use of simple Compton detectors as indicated in the conclusions. Of particular importance to the following, essentially the entire energy spectrum is contained within an energy band ending at about $6 \mathrm{MeV}$.

\section{A. Transport of photons through the cargo container}

For photons with $E_{\gamma} \geq 2 \mathrm{MeV}$, the dominant mode of interaction throughout the periodic table is incoherent scattering. This observation greatly simplifies treatment of the transport of high-energy $\gamma$-rays through the cargo container. The Compton scattering approximation may be applied with negligible error, and the mass attenuation coefficient for photons is then dependent primarily on the ratio of $\mathrm{Z} / \mathrm{A}$. This ratio varies in the range $0.39 \lesssim \mathrm{Z} / \mathrm{A} \lesssim$ 0.5 over most of the periodic table. Thus, the attenuation of high-energy photons will depend primarily on the areal density of a cargo with but weak dependence on its elemental composition. To a fair degree of approximation, the transport of the high-energy $\gamma$ rays of interest may be estimated without undue concern for the makeup of the cargo and its heterogeneity. To be sure, some error will accrue if the cargo contains appreciable quantities of the heaviest elements because of the increasing importance of nucleus-mediated pair production. But this will affect only the highest-energy photons and should not generally result in gross error.

An additional and important simplification that results from restricting attention to the Compton interaction relates to the number of scatterings a photon can suffer before losing so much energy that it is no longer of interest with respect to detection of a unique signature of fission. As shown in Appendix 1, it usually suffices to consider only those photons that suffer fewer than two scatterings in the cargo container before entering into an external 
detector. Because the effects of a single scattering on photon spectra are represented by simple integrals over the cargo container volume, detailed photon transport calculations are generally not required.

When uncollided photons dominate the fission signature, the influence of the cargo material is represented by simple attenuation in the form

$$
\phi_{\gamma}=\frac{S_{0}}{4 \pi r^{2}} \exp (-\langle\Sigma r\rangle) \approx \frac{S_{0}}{4 \pi r^{2}} \exp \left(-4\langle\rho\rangle \frac{r}{100 \mathrm{~cm}}\right)
$$

Here $S_{0}$ is the source strength, $r$ is the source to detector distance and angle brackets represent an average along a given direction. This suggests that that photons escaping from homogeneous cargos will be attenuated by less than two orders of magnitude.

The estimate in eq. (16) neglects the modest dependence of the Compton scattering cross section on photon energy over the energy range of interest. The energy dependence modifies the spectrum of escaping photons because of the decrease in the Compton cross section with increasing photon energy. An estimate of the magnitude of spectrum distortion is made by noting that the Compton scattering cross section for $2 \mathrm{MeV}$ photons is about $5 / 3$ times larger than for $5 \mathrm{MeV}$ photons (eq. A3). This implies that distortion of the delayed $\gamma$-ray spectrum becomes appreciable for cargo thicknesses corresponding to $\left\langle\Sigma\left(E_{\gamma}=2 \mathrm{MeV}\right) r\right\rangle \gtrsim 2$.

\section{ESTIMATES OF THE FISSILE-MATERIAL SIGNATURE}

In this section we show how to use the analytical expressions developed in the previous sections to estimate the delayed $\gamma$-ray signature from fission. We calculate $\epsilon_{\gamma}$, the number of observed high-energy $\beta$-delayed photons per neutron incident on the cargo container. Following the spirit of the preceding sections, we write

$$
\epsilon_{\gamma}=\epsilon_{f} S_{\gamma, 0} e^{-\langle\Sigma r\rangle} \epsilon_{d}
$$

Here $\epsilon_{f}$ represents the number of fissions per source neutron and $S_{\gamma, 0}$ is the number of photons emitted per fission in the time and energy window of interest. For example, if a system can detect photons with energies larger than $2 \mathrm{MeV}$ emitted over a period of $100 \mathrm{sec}$ following fission, then figure 7 gives $S_{\gamma, 0} \approx 0.2$ for ${ }^{235} \mathrm{U}$. In Eq. 17, $\epsilon_{d}$ represents the fraction of escaping high-energy photons that are detected. This clearly depends on characteristics of the photon detector that will not be considered here. It is reasonable to conceive of a 
detector system that will register a substantial fraction of the photons emitted into the solid angle it subtends.

The efficiency for inducing fission depends on characteristics of the fissionable material. If this target material has dimensions that are large relative to the mean free path of a neutron in the fissile material, $\epsilon_{f}$ will scale with the target surface area. If the target is relatively small $\epsilon_{f}$ will instead scale with the target mass. For our present purposes we will suppose that the target is spherical with radius $r_{\text {targ }}$ and completely opaque to thermal neutrons. We also assume that neutrons at higher energies induce fission with 10 percent efficiency. These numbers are appropriate for a target consisting of a $5 \mathrm{~cm}$ sphere of ${ }^{235} \mathrm{U}$. With these assumptions the efficiency for inducing fission can be written

$$
\epsilon_{f} \approx \frac{\pi r_{\mathrm{targ}}^{2}}{\phi_{0} a_{\text {beam }}}\left(\phi^{\mathrm{TH}}(\operatorname{targ})+0.1 \phi^{\mathrm{HE}}(\operatorname{targ})\right)
$$

where $\phi^{\mathrm{TH}}$ and $\phi^{\mathrm{HE}}$ refer to the flux of thermal and all other neutrons at the position of the target, respectively. The factor $\pi$ in eq. (18) should be changed to $4 \pi$ when the (approximately) locally isotropic diffusive flux dominates over the well-collimated uncollided flux.

For homogeneous, non-hydrogenous cargos the uncollided flux at the center of the cargo container along the beam axis is approximately $0.2 \phi_{0}$ for neutrons with incident energy larger than about $5 \mathrm{MeV}$. At a horizontal distance of $1 \mathrm{~m}$ from the beam axis, the total flux is smaller by factors of 4-10. Essentially the entire flux within the container is nonthermal. The total attenuation length for escape of photons arising from a target located at the center of a uniformly filled container is $r \Sigma_{\gamma} \approx 3\left(\rho / 0.5 \mathrm{~g} \mathrm{~cm}^{-3}\right)$. Using these estimates a scaling useful for understanding signal strength from homogeneous non-hydrogenous cargos is obtained as

$$
\frac{\epsilon_{\gamma}}{\epsilon_{d}} \approx 10^{-5} e^{-\left(\left\langle\Sigma_{\gamma} x\right\rangle-3\right)}\left(\frac{\phi_{\text {target }} / \phi_{0}}{0.2}\right)\left(\frac{S_{\gamma, 0}}{0.1}\right)\left(\frac{\pi r_{\text {targ }}^{2} / a_{\text {beam }}}{0.1}\right) .
$$

Implicit in this equation is the assumption that the beam area is larger than the cross sectional area of the fissile material.

The flux in hydrogenous cargos depends strongly on the neutron beam energy and hydrogen density in the cargo. Figure 4 and the equations in section II B can be used for reliable estimates of flux in homogeneous cargos. Note that the flux will usually be dominated by thermal neutrons. 


\section{DISCUSSION AND CONCLUSIONS}

Notwithstanding the real complexities of the wide range of cargos expected in practice, the analyses presented above suggest that for many homogeneous and quasi-homogeneous cargos, the exact nature of the materials is relatively unimportant to estimates of the efficacy of the interrogation scheme. If the cargo is devoid of significant quantities of hydrogenous material, so that thermalization can be neglected, the efficiency for producing fission is not strongly dependent on the elemental composition. When the hydrogenous content is significant, the efficiency for producing fission will depend strongly on the average density of hydrogen and the incident neutron energy. The detailed composition of the hydrogenous material is generally unimportant. Further, significant quantities of high-Z elements will not affect the efficiency to a great extent. For almost all cargo types, the transport of the high-energy delayed $\gamma$ rays through the cargo will be fairly independent of cargo composition other than its mean density. This is true for both homogeneous and heterogeneous cargos.

These observations permit general scoping calculations to estimate the signal incident on an external detector without recourse to detailed transport calculations. As shown in the appendix, the spectrum of photons escaping the cargo will, in many cases be composed of a significant fraction of first-scattered photons that will have energies in the range desired for signal analysis. As pointed out by Norman et al. [2], the natural background radioactivity does not emit energies in excess of $2.6 \mathrm{MeV}$, and this might represent a natural lowerlimit to the energies of detected photons that are used as a signal for fission. To be sure, detector events from cosmic background and subsequent nuclear reactions at higher energies must be considered. But also, the very intense photons at lower energies from fission might suggest an even lower threshold for a fission signal. The exact nature of the lower-limit for a desired signal will depend upon an optimization between the fission-product $\gamma$ rays and the actual background of the environment in which measurements are made. In any event, the large fraction of delayed $\gamma$-ray intensity contained in relatively weak lines suggest that highresolution detectors are not necessary and relatively cheap and simple organic scintillators should suffice in practical applications.

We have not presented a survey of cargos that are non-uniform, or "clumpy". As discussed above and in the first Appendix, photon transport is mostly sensitive to average opacities. Even for clumpy cargos, then simple methods can give good estimates for the spectra of 
photons escaping the container.

Some of the conclusions arrived at above will not be valid for neutrons interrogating very clumpy cargos. One reason is simply that the natural densities of many material are much larger than the allowed maximum mean density of a cargo container. Since the neutron scattering depth for a material of width $x$ is just proportional to $\rho x$, neutrons interrogating clumpy cargos can be attenuated much more than neutrons interrogating homogeneous cargos. For example, a cargo container filled with twenty-seven $(40 \mathrm{~cm})^{3}$ steel cubes on a uniform grid at the nominal density of about $\rho=8 \mathrm{~g} \mathrm{~cm}^{-3}$ has the same mass as a container filled uniformly at a density $\rho=0.6 \mathrm{~g} \mathrm{~cm}^{-3}$. However, the uncollided flux of neutrons passing through two of these cubes is only about $10^{-6}$ of the incident flux. Essentially all of the incident neutrons exit a cube after several scatterings and the diffusive flux will be relatively intense. Both Monte Carlo and analytic calculations show that the diffusive flux can be about two orders of magnitude larger than the uncollided beam flux in a container packed in this way. Clearly, the detailed nature of such "clumped" cargo and the distribution of voids in the container must be considered for field applications.

\section{Acknowledgments}

We are indebted to Dennis Mcnabb for helping us to understand that the absorption length can be much larger than the moderation length in these cargos. We also thank Dennis Slaughter for bringing together the cargo interrogation group and motivating this

project. This work was performed under the auspices of the U.S. Department of Energy by University of California Lawrence Livermore Laboratory under contract W-7405-ENG-48.

\section{APPENDIXES}

\section{APPENDIX A: ESTIMATES OF THE CONTRIBUTION OF MULTIPLY SCAT- TERED PHOTONS TO THE DELAYED $\gamma$-RAY SPECTRUM INCIDENT UPON AN EXTERNAL DETECTOR}

The influence of Compton scattering on the spectrum of $\beta$-delayed $\gamma$-rays incident on an external detector can be estimated by considering the case of an isotropic mono-energetic 
photon source surrounded by some attenuating material. The probability that an emitted photon arrives at a detector outside the cargo container without having previously scattered is

$$
P_{0} \approx \frac{A_{d}}{4 \pi d^{2}} e^{-a / \lambda}
$$

Here $A_{d}$ is the detector area, $d$ is the source to detector distance, $a$ is the path length through the attenuating medium, and $\lambda$ is the mean free path of photons in the medium. In the limit where only Compton interactions are significant, reasonable for the range of photon energies considered here,

$$
\lambda^{-1}=\rho\langle Z / A\rangle N_{a} \sigma_{c} \approx(5 \mathrm{~cm})^{-1}\left(\frac{\langle Z / A\rangle}{0.5}\right)\left(\frac{\rho}{1 \mathrm{~g} \mathrm{~cm}^{-3}}\right)\left(\frac{\sigma_{\mathrm{c}}}{\sigma_{T}}\right) .
$$

Here $\rho$ is the density of scattering material, $\langle Z \mid A\rangle$ is the average charge to mass ratio of the material, $\sigma_{T}$ is the Thomson cross section, and

$$
\sigma_{\mathrm{c}} \approx \frac{3}{4} \sigma_{T} \frac{m_{e} c^{2}}{E_{\gamma}}\left(\frac{1}{4}+\frac{1}{2} \ln \left(\frac{2 E_{\gamma}}{m_{e} c^{2}}\right)\right)
$$

is the total Compton scattering cross section.

Photons which scatter once in the cargo container before escaping have energies $E_{\gamma}^{\prime}$ given by the familiar relation

$$
E_{\gamma}^{\prime}=\frac{E_{\gamma}}{1+\frac{E_{\gamma}}{m_{e} c^{2}}(1-\cos \theta)} .
$$

Here $\theta$ is the scattering angle of the photon and $m_{e} c^{2}$ is the rest energy of an electron. Because of the limited energy range of the detected photons that are useful for extracting a signal from decay of fission fragments, scattering by an angle greater than some $\theta_{\max }$ results in a photon with energy too small to be interesting. This maximum scattering angle is related to the largest acceptable ratio $\alpha_{e} \equiv\left[E_{\gamma} / E_{\gamma}^{\prime}-1\right]$ by

$$
\frac{\left[E_{\gamma} / E_{\gamma}^{\prime}-1\right]_{\max }}{\left(E_{\gamma} / m_{e} c^{2}\right)} \equiv \frac{\alpha_{e}}{\left(E_{\gamma} / m_{e} c^{2}\right)}=1-\cos \theta_{\max }
$$

For cargo applications $\alpha_{e}$ is of order unity. For example, if the typical interesting photon has an energy of $3 \mathrm{MeV}$, and the lowest limit of the detected photon signal that is of interest is $2 \mathrm{MeV}$, then $\alpha_{e}=1 / 2$.

The probability $P_{1}$ that a photon arrives at the detector after suffering one small angle $\left(\theta<\theta_{\max }\right)$ scattering is readily calculated for a given cargo loading scenario from

$$
P_{1}=\int_{\theta<\theta_{\max }} \exp \left(-n_{\lambda}\right) \frac{1}{\sigma_{\mathrm{c}}} \frac{d \sigma}{d \Omega} \frac{A_{d}}{r_{2}^{2}} \frac{d^{3} x}{4 \pi r^{2} \lambda} .
$$


Here $r$ is the distance from the photon source to the volume element $d^{3} x$ where a scattering takes place, $r_{2}$ is the distance from that volume element to the detector and $n_{\lambda}$ is the total number of mean free paths the photon must traverse to arrive at the detector. Because $\theta_{\max }$ is small for our applications, the differential scattering cross section appearing in Eq. (A6) is fairly approximated by the zero degree forward scattering differential cross section. This implies that the fraction of scatterings resulting in a small-angle $\left(\theta<\theta_{\max }\right)$ collision is

$$
\frac{1}{\sigma_{c}}\left(\frac{d \sigma}{d \Omega}(\theta \approx 0)\right) d \Omega\left(\theta<\theta_{\max }\right) \approx \frac{2 \alpha_{e}}{3}
$$

For a point photon source embedded in a uniform cargo container this implies

$$
\frac{P_{1}}{P_{0}} \approx(2 / 3) \alpha_{e} \Sigma r
$$

In general, once scattered photons are expected to dominate the signal once $\Sigma r$ is larger than about three.

\section{APPENDIX B: DIFFUSION KERNEL FOR A RECTANGULAR BOX}

Diffusion calculations describing transport in finite systems often make the simplifying approximation that the flux falls to zero at an extrapolation distance $d_{e x}=2 / 3 \Sigma$ from the system walls. With this assumption the diffusion kernel for homogeneously filled cargo containers can be represented by

$$
G_{\text {fin }}=\frac{4}{W^{2}} \sum_{n, m=1} \frac{\sin (\tilde{y} m) \sin \left(\tilde{y}^{\prime} m\right) \sin (\tilde{z} n) \sin \left(\tilde{z^{\prime}} n\right) \sinh \left(\gamma_{m n} x_{<}\right) \sinh \left(\gamma_{n m}\left(L-x_{>}\right)\right)}{\gamma_{n m} \sinh \left(\gamma_{n m} L\right)}
$$

where $\tilde{a} \equiv a \pi / W, \gamma_{n m}=(1 / W) \sqrt{m^{2}+n^{2}}, x_{<}=\min \left(x, x^{\prime}\right)$, and $x_{>}=\max \left(x, x^{\prime}\right)$. This equation assumes that the cargo container has one corner at the coordinate system origin and occupies only the positive octant of this system. The long axis of the container is assumed to be along the $x$-axis. The length of the container along this direction is $L-d_{e x}$, while the container length along the other two directions is $W-d_{e x}$.

Eq. B1 points to an interesting and sometimes counter-intuitive aspect of neutron diffusion in finite systems. Namely, $G_{\text {fin }}$ depends only weakly (through the definition of $L$ and $W$ ) on the neutron mean free path. This means that the relative error induced by use of the infinite space kernel $G_{\text {inf }} \propto 1 / r$ (rather than the more appropriate $G_{\text {fin }}$ ) is approximately 
the same whether the neutron mean free path is $30 \mathrm{~cm}$ or $30 \mathrm{~mm}$.

[1] Slaughter, D., Accatino, M., Bernstein, A., Candy, J., Dougan, A., Hall, J., Loshak, A., Manatt, D., Meyer, A., Pohl, B., Prussin, S.G., Walling, R., Weirup, D., 2003, Lawrence Livermore Laboratory Report, UCRL-ID-155315

[2] Norman, E.B., Prussin, S.G., Larimer, R.-M., Shugart, H., Browne, E., Smith, A., McDonald, R.J., Nitsche, H., Gupta, P. Frank, M., Gosnell, T., 2004, NIM A, 521, 608

[3] Wilcox, T.P. \& Lent, E., 1989, Lawrence Livermore Laboratory Report, M-221-1

[4] Weinberg, A.M. \& Wigner, E.P. 1958, The Physical Therory of Neutron Chain Reactors, University of Chicago Press

[5] Argonne National Laboratory, July 1963, Reactor Physics Constants, United States Atomic Energy Commission

[6] Pruet, J., Hall, J., Descalle, M.-A. \& Prussin, S.G. 2004, NIMB 222, 403.

[7] Norman, E.B. et al. 2004, in preparation

[8] England, T.R. \& Rider, B.F. 1994, LA-UR-94-3106, ENDF-349

[9] Kinsey, R.R., Dunford, C.L., Tuli, J.K. \& Burrows, T.W., 1997, Capture Gamma-Ray Spectroscopy and Related Topics, Vol. 2., 657

[10] Although other light nuclides such as deuterium and ${ }^{9} \mathrm{Be}$ can be good moderators, they are not normally found as major components of international commerce.

[11] http://t2.lanl.gov

This work was performed under the auspices of the U. S. Department of Energy by University of California, Lawrence Livermore National Laboratory under contract W-7405-Eng-48. 


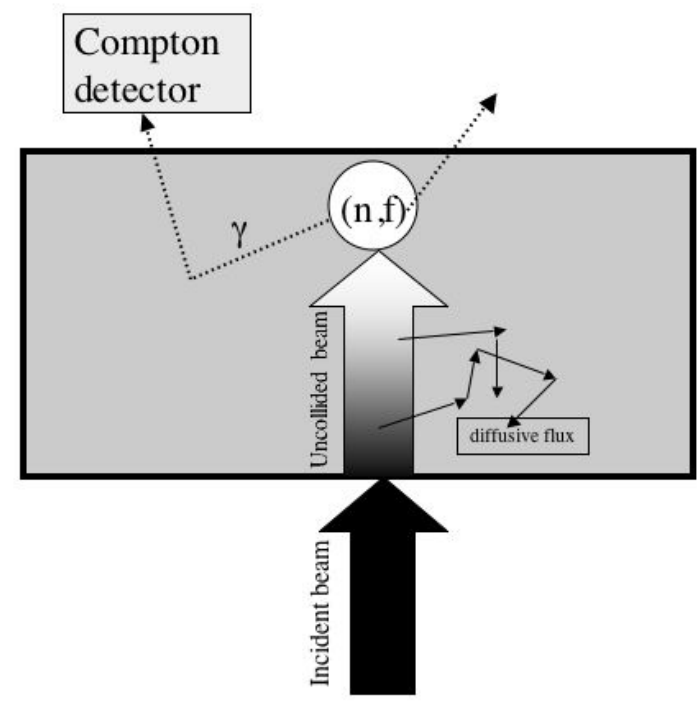

FIG. 1: Schematic illustration of the neutron interrogation system considered here. 


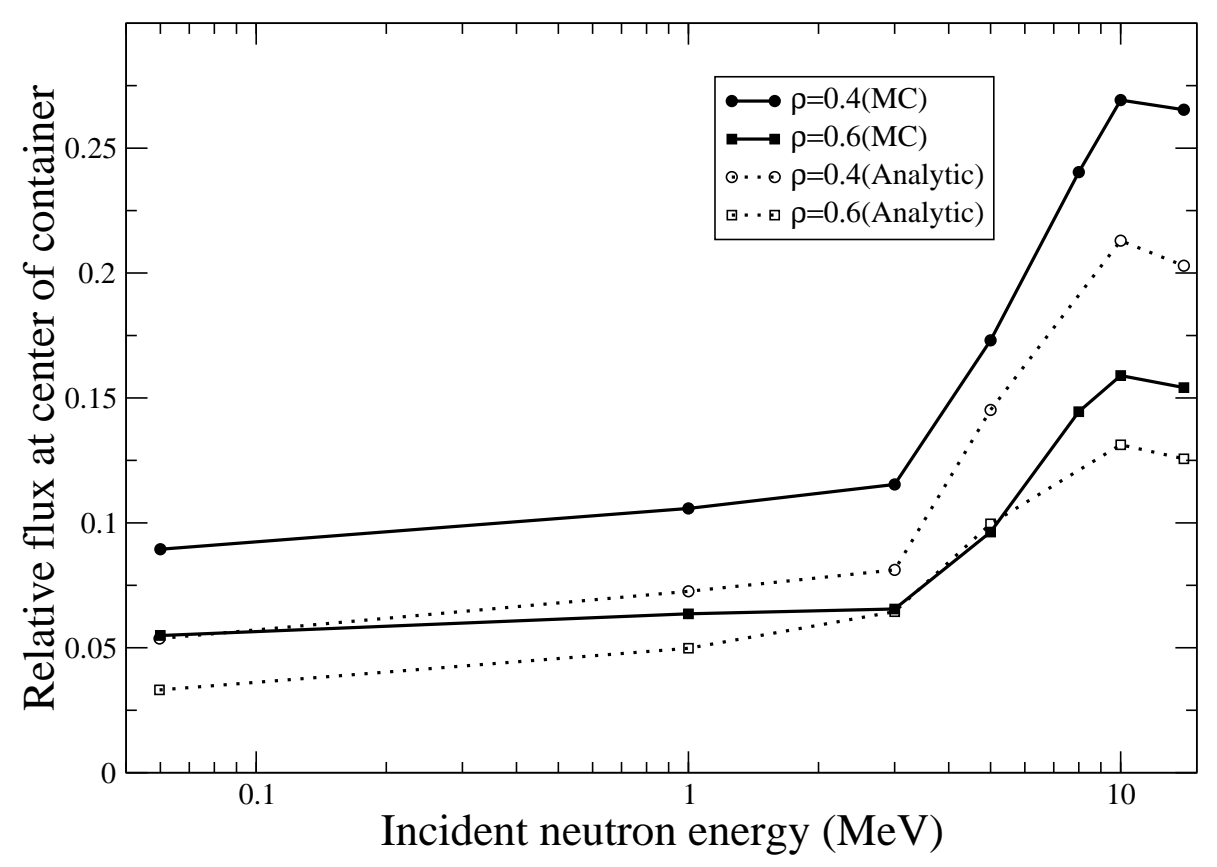

FIG. 2: The flux at the center of a cargo container filled homogeneously with aluminum as a function of neutron source energy and mass density. A neutron beam of unit flux is incident normal to the floor of the container and has a $15 \mathrm{~cm}$ radius. Results of Monte Carlo calculations are labelled with (MC), while estimates based on the sum of eq. (9) and eq. (6) are labeled with (Analytic). For the analytical calculations, neutrons scattering in the 3 -cm thick wood floor covering are assumed lost from the system. This is overly conservative and results in an underestimate of the flux for low neutron source energies (see text). Sampling uncertainties in the Monte Carlo runs are approximately $0.1 \%$ near the center of the container and approximately $1 \%$ near the edge of the container. 


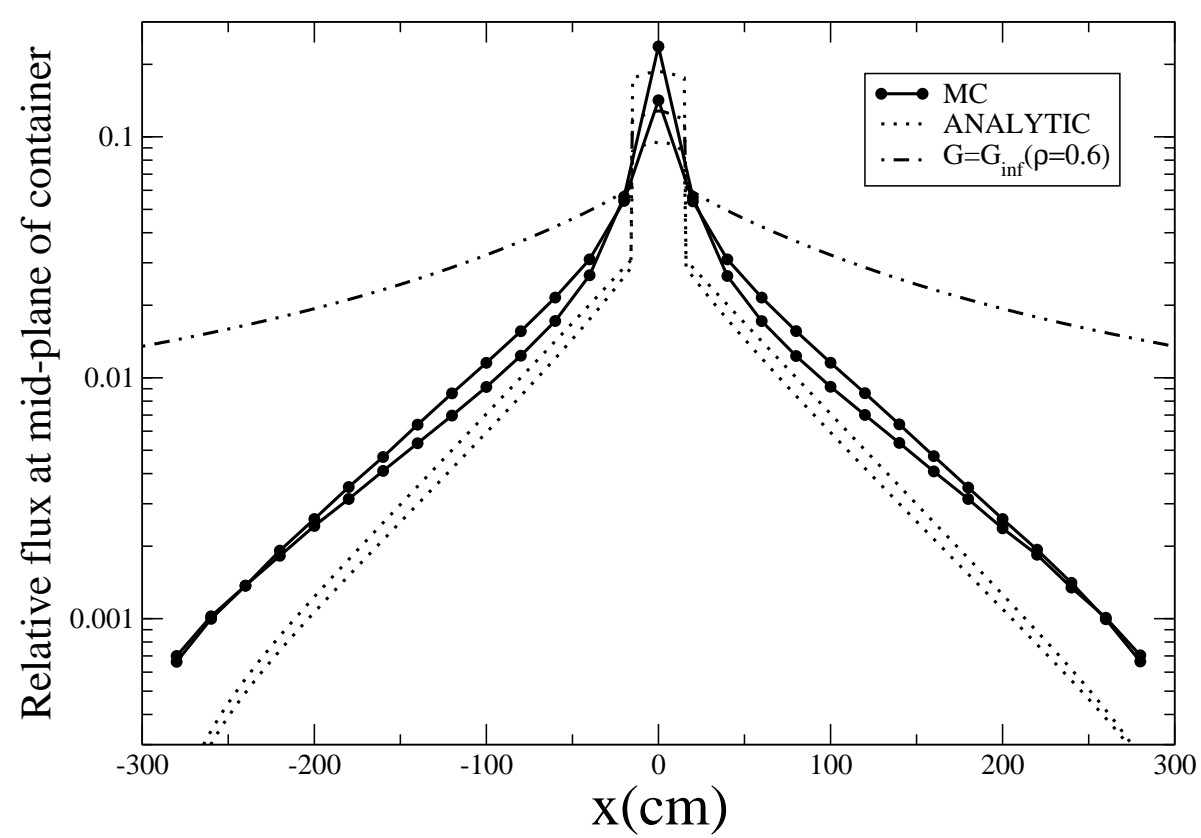

FIG. 3: The flux along the horizontal centerline at the mid-pale of a cargo container filled uniformly with aluminum. The incident beam used for these calculations is comprised of mono-energetic $14 \mathrm{MeV}$ neutrons with the same characteristics as the beam described in figure 2 . Here $x$ measures the distance along the long (20 foot) axis of the cargo container. The upper and lower lines labeled MC and ANALYTIC represent flux in a container filled at $\rho=0.4 \mathrm{~g} / \mathrm{cm}^{3}$, while the lower lines labeled MC and ANALYTIC represent flux in a container filled at $\rho=0.6 \mathrm{~g} / \mathrm{cm}^{3}$. The line labeled $G_{\text {inf }}$ represents flux in the absence of neutron leakage (i.e., under the assumption that the cargo container has infinite size). Note that at distances from the beam larger than about $100 \mathrm{~cm}$ the infinite-space approximation grossly overestimates the flux. Sampling uncertainties in the Monte Carlo runs are approximately $0.2 \%$ near the center of the container and approximately $50 \%$ near the edge of the container. 


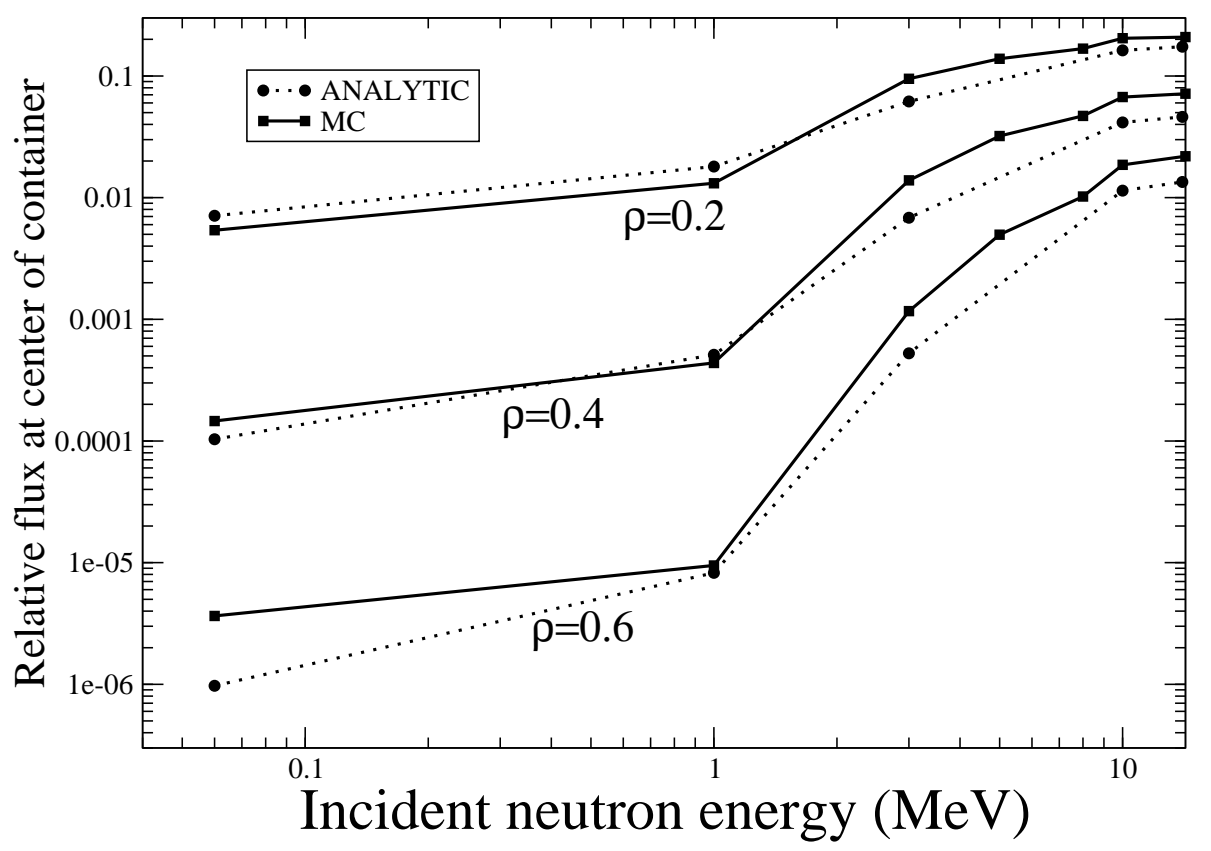

FIG. 4: The flux at the center of a cargo container filled homogeneously with wood (see text) as a function of neutron source energy and mass density. Neutron beam characteristics used for this calculation are the same as those described in the caption of figure 2. 


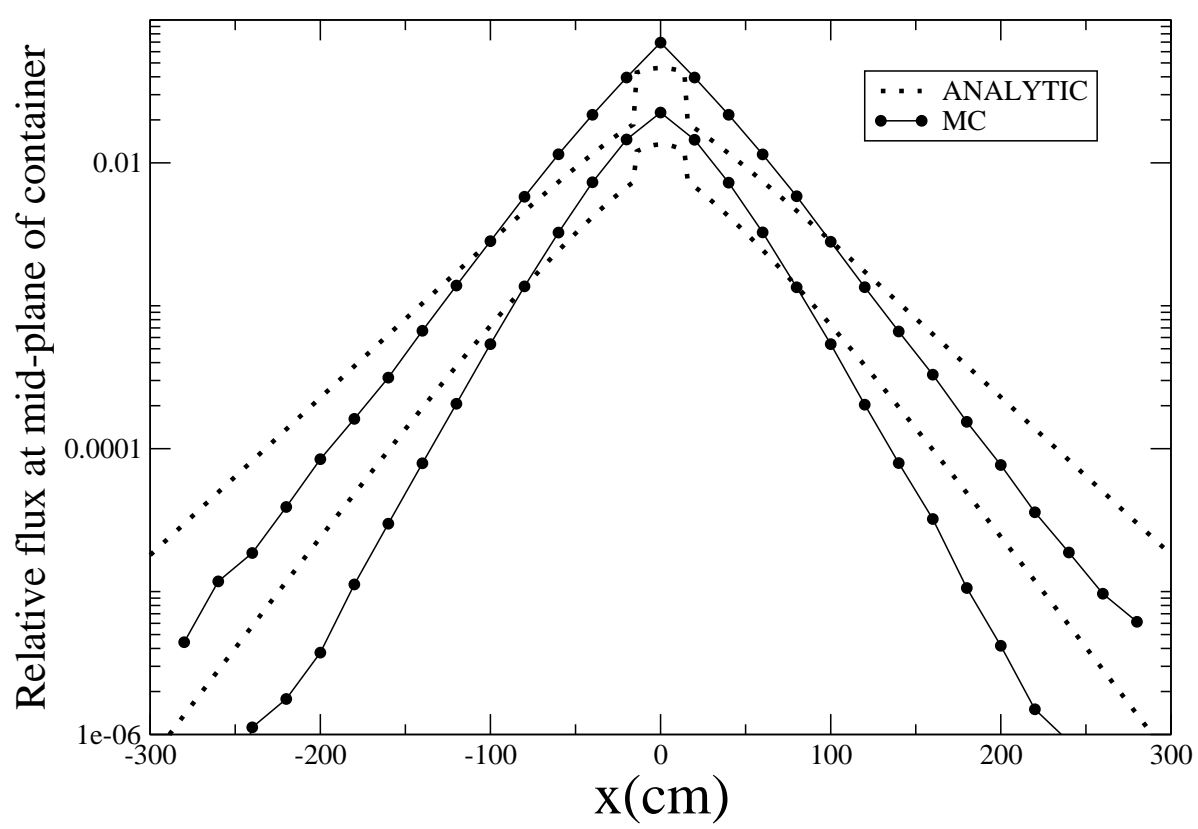

FIG. 5: Same as figure 3 except for a container filled uniformly with plywood. The upper and lower lines represent flux in a container filled at $\rho=0.4 \mathrm{~g} / \mathrm{cm}^{3} \rho=0.6 \mathrm{~g} / \mathrm{cm}^{3}$, respectively. 


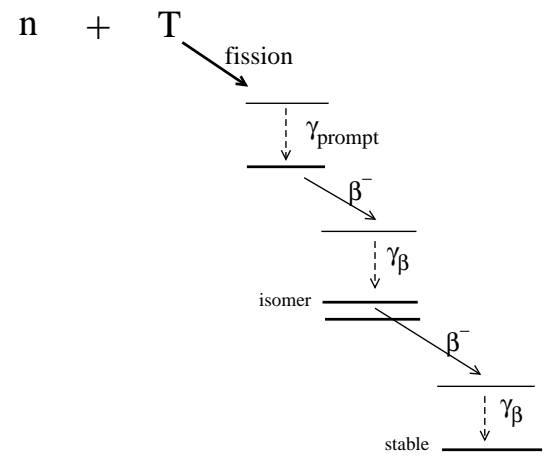

FIG. 6: Illustration of some processes leading to the production of post-fission $\beta$-delayed photons. 


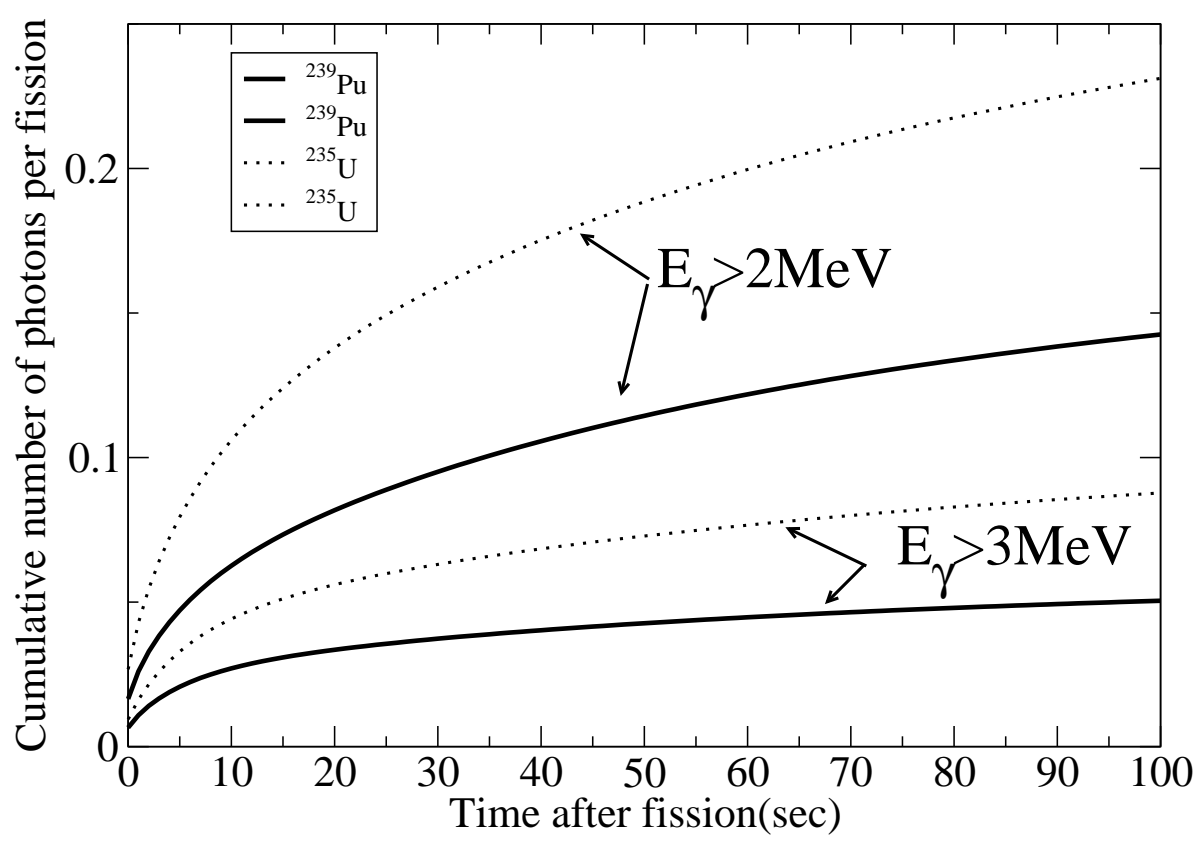

FIG. 7: Estimate of the cumulative number of high-energy photons produced following thermal neutron induced fission of ${ }^{235} \mathrm{U}$ and ${ }^{239} \mathrm{Pu}$. 


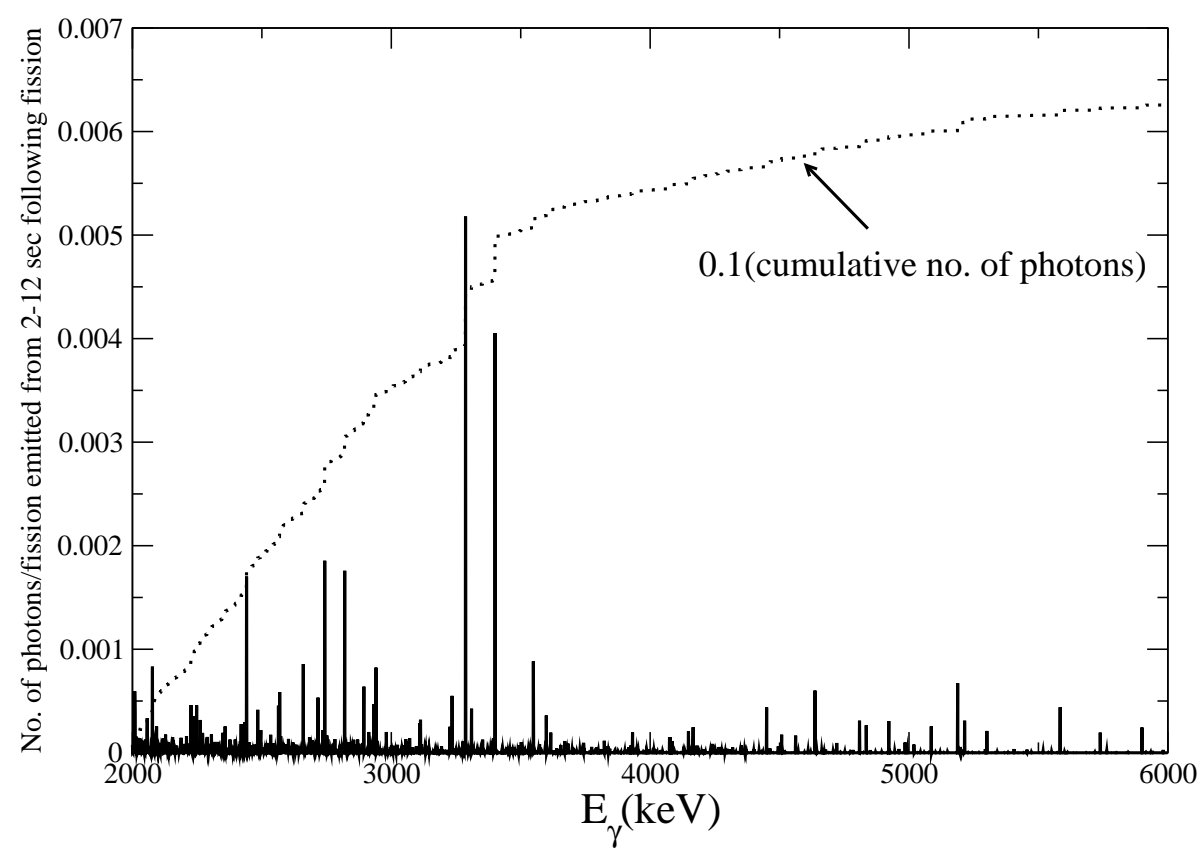

FIG. 8: Estimate of the spectrum of photons emitted in the time interval from two to twelve seconds following thermal neutron induced fission of ${ }^{235} \mathrm{U}$. The dotted line is one tenth the cumulative sum of the individual lines. 
TABLE I: Some quanitities useful for calculations involving neutron transport in hydrogenous materials

\begin{tabular}{lcccc}
\hline \hline Material & $\mathrm{E}_{\mathrm{n}}(\mathrm{MeV})^{a}$ & $\tau\left(\mathrm{cm}^{2}\right)^{b}$ & $\lambda_{0}(\mathrm{~cm})^{c}$ & $k^{-1}(\mathrm{~cm})^{d}$ \\
\hline Plywood & 0.06 & 30 & 2.2 & 7.8 \\
Plywood & 1 & 70 & 5 & 7.8 \\
Plywood & 3 & 185 & 10 & 7.8 \\
Plywood & 10 & 700 & 19 & 7.8 \\
Plywood & 14 & 800 & 20 & 7.8 \\
\hline \hline
\end{tabular}

Values listed here assume a plywood density of $0.6 \mathrm{~g} / \mathrm{cm}^{3}$.

${ }^{a}$ Neutron energy.

${ }^{b}$ Fermi age of thermal neutrons with the given incident energy. Note that tau scales with density as $\tau \propto \rho^{-2}$.

${ }^{c}$ Mean free path of neutrons with the given incident energy. Note that $\lambda \propto \rho^{-1}$.

${ }^{d}$ Diffusion length of thermal neutrons. Note that $k^{-1} \propto \rho^{-1}$ 